Article

\title{
Evaluation of Antioxidant Activities of Aqueous Extracts and Fractionation of Different Parts of Elsholtzia ciliata
}

Xiangping Liu ${ }^{1,2, \dagger}$, Jia Jia ${ }^{3, \dagger}$, Lei Yang ${ }^{1, *}$, Fengjian Yang ${ }^{1}$, Hongshuang Ge ${ }^{1}$, Chunjian Zhao ${ }^{1}$, Lin Zhang ${ }^{1}$ and Yuangang $\mathrm{Zu}{ }^{1}{ }^{1, *}$

1 State Engineering Laboratory for Bioresource Eco-Utilization, Northeast Forestry University, Harbin 150040, China; E-Mails: 1xp110@hotmail.com (X.L.); yangfj@nefu.edu.cn (F.Y.); swx05131ghs@126.com (H.G.); zcjsj@163.com (C.Z.); zhanglin6600@sina.com (L.Z.)

2 College of Animal Science \& Veterinary Medicine, Heilongjiang Bayi Agricultural University, Daqing 163319, China

3 Preclinical Medicine Department of Daqing Medical College, Daqing 163319, China; E-Mail: jiajiaplay2006@163.com (J.J.)

$\dagger$ These authors contributed equally to this work.

* Authors to whom correspondence should be addressed; E-Mails: ylnefu@163.com (L.Y.); zygnefu@163.com (Y.Z.); Tel.: +86-451-8219-1387 (Y.Z.); Fax: +86-451-8210-2082 (Y.Z.).

Received: 17 April 2012; in revised form: 25 April 2012 / Accepted: 28 April 2012 /

Published: 9 May 2012

\begin{abstract}
The aim of this study was to investigate the antioxidant and free-radical scavenging activity of extract and fractions from various parts of Elsholtzia ciliata. The inflorescences, leaves, stems and roots of E. ciliata were extracted separately and two phenolic component enrichment methods: ethyl acetate-water liquid-liquid extraction and macroporous resin adsorption-desorption, were adopted in this study. The antioxidant activities of water extracts and fractions of E. ciliata were examined using different assay model systems in vitro. The fraction root E (purified by HPD300 macroporous resin) exhibited the highest total phenolics content (497.2 $\pm 24.9 \mathrm{mg} \mathrm{GAE} / \mathrm{g})$, accompanied with the highest antioxidant activity against various antioxidant systems in vitro compared to other fractions. On the basis of the results obtained, E. ciliata extracts can be used potentially as a ready accessible and valuable bioactive source of natural antioxidants.
\end{abstract}

Keywords: Elsholtzia ciliata; free-radical scavenging activity; antioxidant activity; phenolics content 


\section{Introduction}

The common free radicals are oxygen-reactive species (ROS), which are formed during natural metabolism and are in dynamic balance between their biosynthesis and their removal by antioxidant systems in human bodies. When the mechanism of antioxidant protection becomes unbalanced by factors such as ageing, deterioration of physiological functions may occur, resulting in oxidative stress and thus can lead to cell injury and various relevant diseases such as cardiovascular diseases, cancer and accelerated ageing [1-3]. However, the innate defense systems of body may be supported by antioxidative compounds taken as foods, cosmetics and medicine. Although the most widely used antioxidants are synthetic antioxidants, such as butylated hydroxytoluene (BHT) and butylated hydroxyanisole (BHA), their toxic properties and unwanted side effects limit their widespread use [4-7]. Hence, the development of alternative antioxidants from natural sources has considerable prospects.

It is common knowledge that naturally occurring substances in higher plants have antioxidant activity. In the recent years, more attention has been paid to the protective biochemical functions of naturally occurring antioxidants in the cells of the organisms containing them [8-13].

There has been a growing interest in Elsholtzia species because of its long history for over thousands of years. The studies of Elsholtzia species are mainly focused in constituents of essential oil [14-16], antivirus function [17] and so on. Elsholtzia ciliata, a plant which is also known as "Xiang Ru" in China, is an annual herb that belongs to the Elsholtzia species that is widely distributed throughout China, Korea and Europe. The seeds of E. ciliata are powdered and used for flavoring foods. The chemical components of the crude drug are essential oil, elsholtzia ketone, flavonoids, steroids [18] and triterpenes. As a favorite and widely used medical herb in traditional Chinese medicine (TCM), the herb has been used for the treatment of fever, headache, diarrhea and edema [19]. Moreover, E. ciliata has been officially listed in the menu of edible herbs by the Ministry of Health of China.

Researchers have sought to isolate powerful and nontoxic natural antioxidants from edible plants not only to prevent autoxidation and lipid peroxidation, but recently also to replace synthetic antioxidants. E. ciliata has been more often used as a food than for medicinal purposes. To our knowledge, there is no report in the literature on the antioxidant potential of the whole plant. The main objectives of this work were to determine the phenolics content, evaluate the antioxidant activities of aqueous extracts and fractionation from different parts of E. ciliata by two different enrichment methods: ethyl acetate-water liquid-liquid extraction and macroporous resin adsorption-desorption, were adopted.

\section{Results and Discussion}

\subsection{Extraction Yield and Total Phenolics Content of Different Parts of E. ciliata Plant}

The amount of materials that can be extracted from a plant depends on the vigour of the extraction procedure and the possibility also exists of sample-to-sample variation in the extracted material. The isolation procedure for extracts of E. ciliata plant is shown in Figure 1, the results on the yields of extraction are summarized in Table 1, and the total phenolics contents of E. ciliata plant parts are shown in Figure 2. The crude water extract yield of all plant parts varied from $30.64 \pm 1.04$ to 
$5.60 \pm 0.16 \mathrm{~g} / 100 \mathrm{~g}$ dry raw materials. Among all the plant parts, the crude water extract of leaf obtained the highest extraction yield $(30.64 \pm 1.04 \mathrm{~g})$, while the root yielded the lowest $(5.60 \pm 0.16 \mathrm{~g})$. The yield of all the fractions is presented in different order.

Figure 1. Isolation procedure for extracts of E. ciliata plant parts.

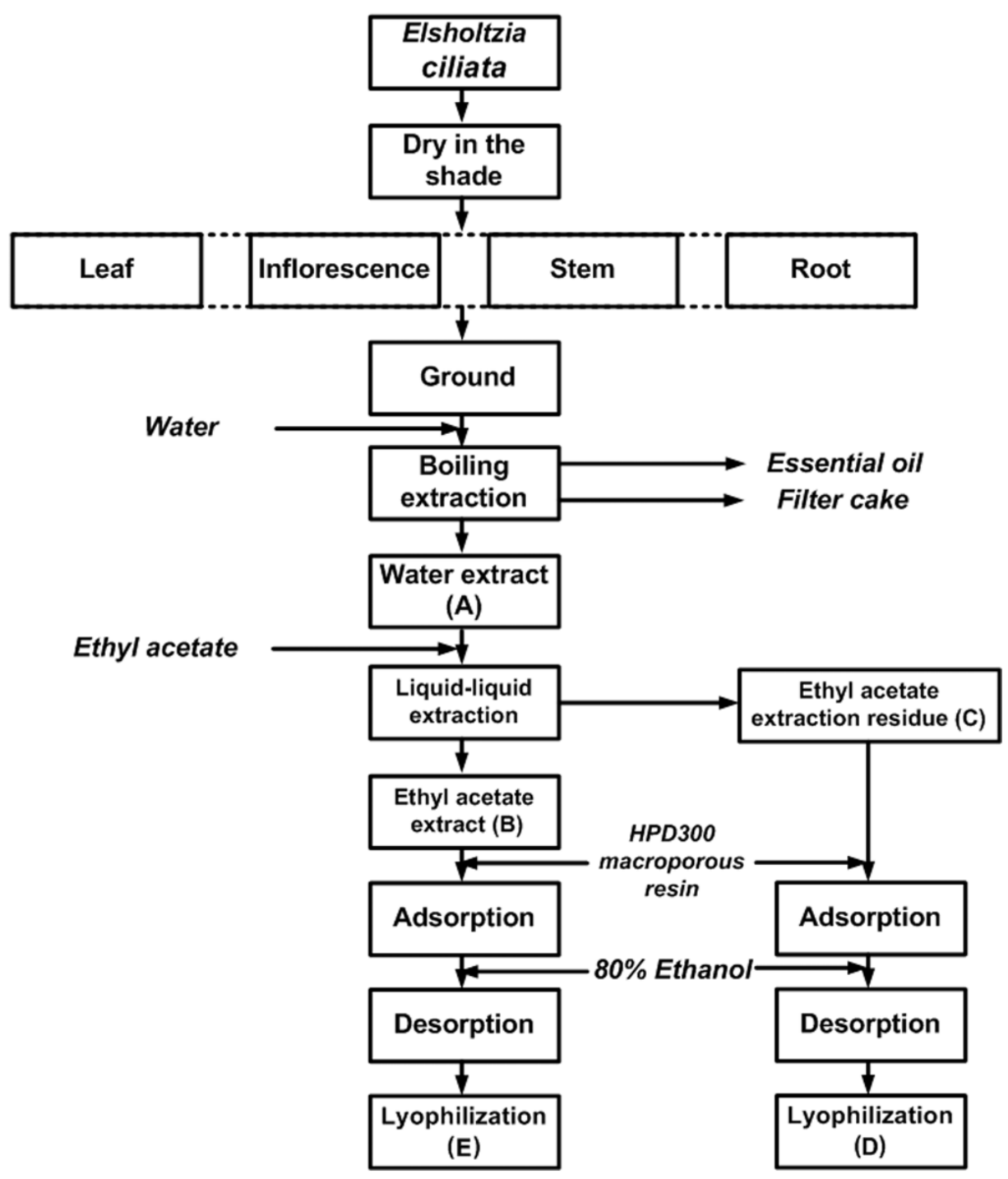

Table 1. Yields of extraction of E. ciliata in different plant parts.

\begin{tabular}{ccccc}
\hline Yield & Leaf (g) & Inflorescence (g) & Stem (g) & Root (g) \\
\hline $\mathrm{A}^{\mathrm{a}}$ & $30.64 \pm 1.04$ & $15.26 \pm 0.34$ & $10.56 \pm 0.43$ & $5.60 \pm 0.16$ \\
$\mathrm{~B}^{\mathrm{b}}$ & $2.38 \pm 0.09$ & $2.49 \pm 0.09$ & $2.49 \pm 0.09$ & $1.41 \pm 0.05$ \\
$\mathrm{C}^{\mathrm{b}}$ & $2.39 \pm 0.07$ & $2.28 \pm 0.09$ & $2.29 \pm 0.06$ & $3.49 \pm 0.09$ \\
$\mathrm{D}^{\mathrm{c}}$ & $0.13 \pm 0.01$ & $0.09 \pm 0.01$ & $0.12 \pm 0.01$ & $0.29 \pm 0.01$ \\
$\mathrm{E}^{\mathrm{c}}$ & $0.54 \pm 0.02$ & $0.48 \pm 0.01$ & $0.65 \pm 0.02$ & $0.20 \pm 0.01$ \\
\hline
\end{tabular}

${ }^{\mathrm{a}}$ From $100 \mathrm{~g}$ dry raw materials; ${ }^{\mathrm{b}}$ From $5 \mathrm{~g} \mathrm{~A} ;{ }^{\mathrm{c}}$ From $1 \mathrm{~g} \mathrm{~B}$ or C; A, B, C, D and E, the same as Figure 1. 
Figure 2. Total phenolics in fractions of different parts of E. ciliate.

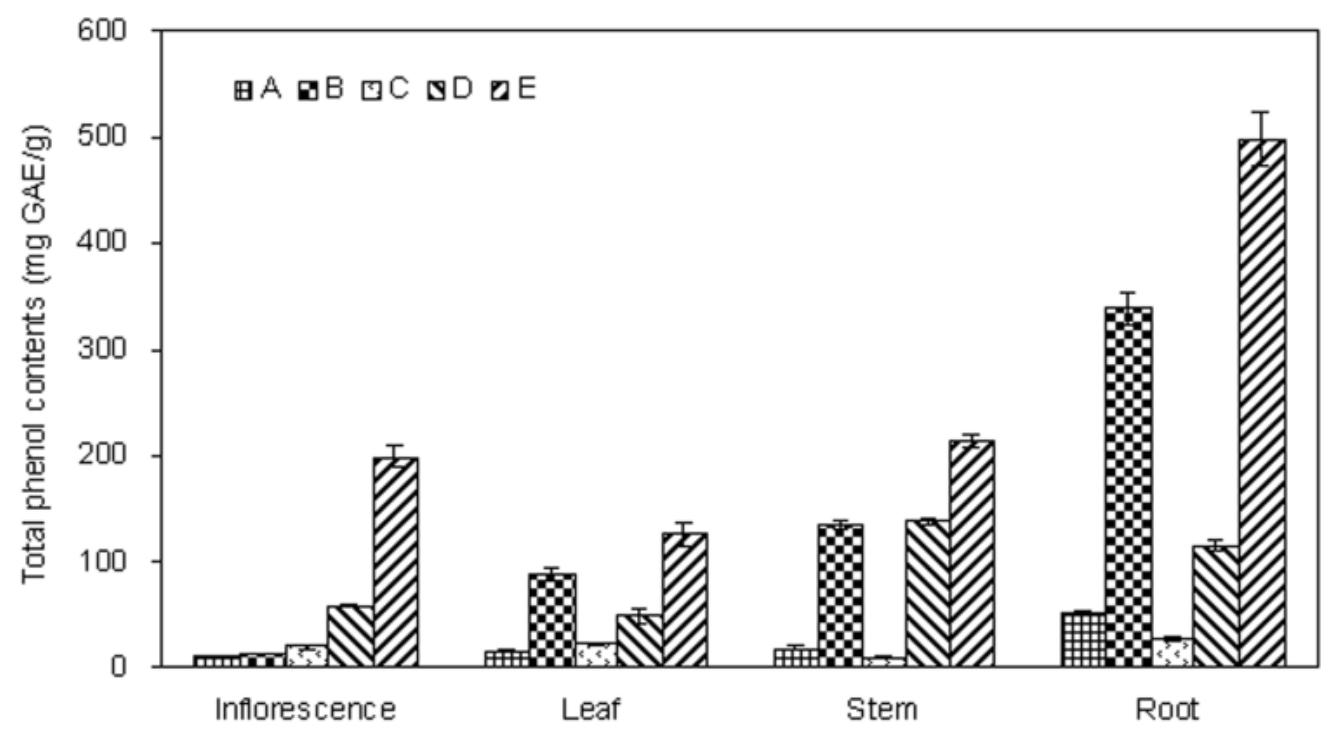

Many studies have pointed out that there is a positive correlation between antioxidant activity potential and amount of phenolic compounds of the extracts [20-22]. Therefore, the amount of total phenolics in the extracts was determined in this study. The total phenolics content of the E. ciliata extracts was determined through a linear gallic acid standard curve $\left(y=0.0039 x-0.009 ; R^{2}=0.9994\right)$ and expressed as milligram of gallic acid equivalents per gram of extract (mg GAE/g). As can be seen from Figure 3, the total phenolics content was varying in different extracts of E. ciliata plant. The amount of the total phenolics was significantly higher in root $\mathrm{E}(497.2 \pm 24.9 \mathrm{mg} \mathrm{GAE} / \mathrm{g})$ compared with the other parts of plant, and followed by stem E $(213.1 \pm 6.2 \mathrm{mg}$ GAE/g), inflorescence E $(198.2 \pm 10.1 \mathrm{mg} \mathrm{GAE} / \mathrm{g})$. Fraction E contained the highest phenolics content among all five fractions. Furthermore, fraction B was found to be the second one, followed by fraction E. This result suggested that the phenolic compounds might selectively be extracted into the ethyl acetate fraction from E. ciliata and be further enriched by macroporous resin technology, but suitable adjustment of the extraction parameters of process is required.

\subsection{Scavenging Effect on DPPH Radical}

The ability of fractions from E. ciliata to quench reactive species by hydrogen donation was measured through the DPPH radical scavenging activity assay [23,24]. As a kind of stable free radical, DPPH can accept an electron or hydrogen radical to become a stable diamagnetic molecule, which is widely used to investigate radical scavenging activity [25]. The analysis of DPPH scavenging activity $(\mathrm{SC} \%)$ are presented in Figure 3, and the $\mathrm{EC}_{50}$ was calculated by the regression analysis formulas under graph. We observed that, different distracts in different parts of E. ciliata plant showed a concentration-dependent DPPH radical scavenging activity. The five fractions at each sequential step exhibited varying degrees of scavenging capacities. On the whole, the activity of the fractions towards the DPPH radical increased in the order $\mathrm{C}<\mathrm{A}<\mathrm{D}<\mathrm{B}<\mathrm{E}$. Among the five fractions, fraction $\mathrm{E}$ exhibited the highest scavenging activity with the lowest $\mathrm{EC}_{50}$ value of $0.97,0.57,1.03,0.09 \mathrm{mg} / \mathrm{mL}$ in leaf, stem, inflorescence and root, respectively, which is comparable and even lower to that of BHT (0.45), BHA (0.21), and $\mathrm{Vc}(0.41)$. Especially the root fractionE showed significantly stronger 
scavenging potency than that of other parts of plant, which is followed by fraction B. This result suggested that some components within ethyl acetate fractions were strong radical-scavenging components. On the other hand, fractions $\mathrm{E}$ and $\mathrm{D}$ were superior to fractions $\mathrm{B}$ and $\mathrm{D}$, respectively, which proved that elution process of HPD300 macroporous resin by $80 \%$ ethanol also has a remarkable effect to concentrate radical-scavenging components.

Figure 3. DPPH radical scavenging activity of sequential extracts in different parts of E. ciliata. Each value is the mean of triplicate measurements; Concentrations relative to Scavenging activity (SC\%); Regression analysis formulas were listed under each graph.
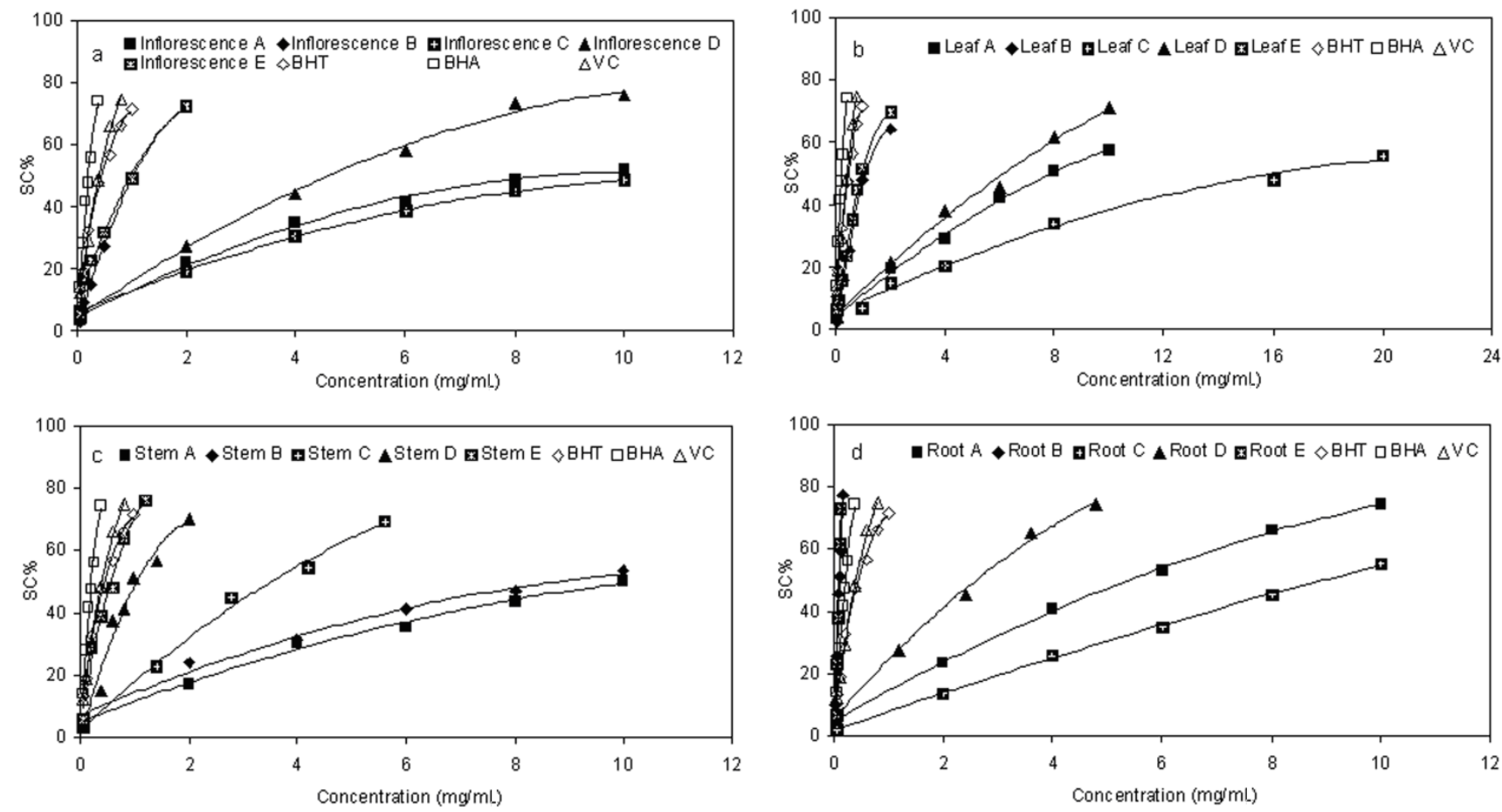

\subsection{Reducing Power}

The potassium ferricyanide reduction method was used to evaluate the reducing power of plant phenolics, which is a widely used method in measuring antioxidant activity of phenolic compounds. In this assay, the antioxidants present in the test solution can reduce the $\mathrm{Fe}^{3+} /$ ferricyanide complex to the ferrous form by donating an electron. Increasing absorbance of the reaction mixture at $700 \mathrm{~nm}$ indicates an increase in the reducing power [26]. In the present study, as shown in Figure 4, the reducing power of the five fractions exhibited a similar concentration-dependent activity pattern as given in the DPPH analysis. Among all fractions, inflorescence fraction E $(0.937 \pm 0.024$ at $0.1 \mathrm{mg} / \mathrm{mL})$ (Figure $4 \mathrm{a})$, root B $(0.817 \pm 0.027$ at $0.1 \mathrm{mg} / \mathrm{mL})$ and root $\mathrm{E}(0.987 \pm 0.019$ at $0.1 \mathrm{mg} / \mathrm{mL})$ (Figure $4 \mathrm{~d}$ ) were found to be superior to the other fractions, but significantly lower than the positive control BHT $(0.423 \pm 0.011$ at $0.1 \mathrm{mg} / \mathrm{mL})$, BHA $(0.580 \pm 0.015$ at $0.1 \mathrm{mg} / \mathrm{mL})$ and comparable with $\mathrm{Vc}(0.754 \pm 0.011$ at $0.1 \mathrm{mg} / \mathrm{mL}$ ). Fractions $\mathrm{C}$ and A possessed weaker activity in all four parts for this test system. Meanwhile, fractions $\mathrm{E}$ were the strongest radical reducer, compared with the others. However, there were differences in the results among fractions $\mathrm{D}$ of different parts of plant. This is comparable with fractions $\mathrm{A}$ and $\mathrm{C}$ for leaf and root, which are different The main objectives of this work were to 
determine the phenolics content, evaluate the antioxidant activities of aqueous extracts and fractionation from different parts of E. ciliata by two different enrichment methods, ethyl acetate-water liquid liquid extraction and macroporous resin adsorption - desorption, were adopted.

Figure 4. Reducing Power for Fractions of Different Parts of E. ciliata. Concentrations Relative to Absorbance and Each Value are the Mean of Triplicate Measurements.
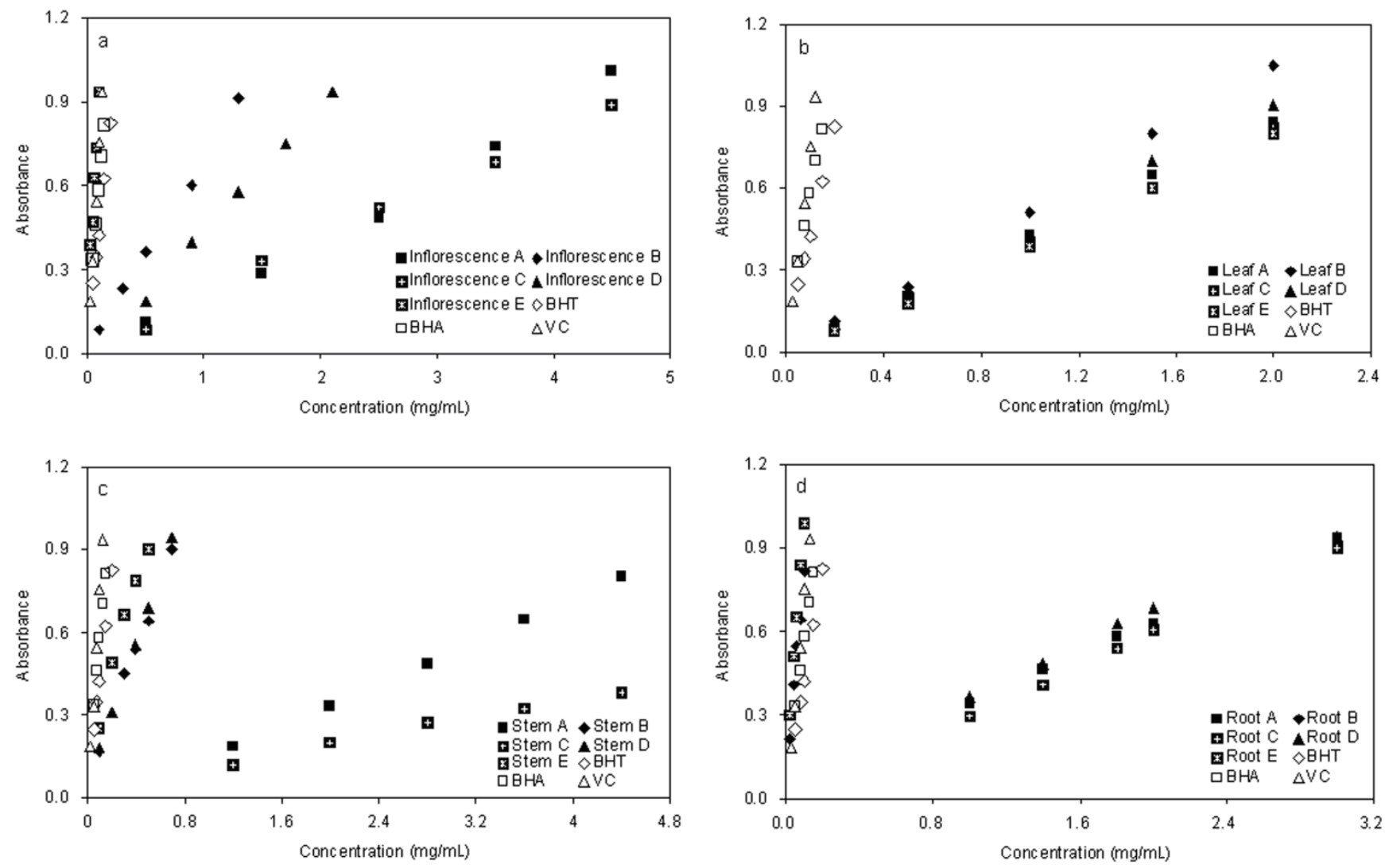

\subsection{Ferric-reducing Antioxidant Power (FRAP)}

FRAP is a simple direct test for measuring antioxidant activity by quenching ferric reducing capacity [8], which was used in many studies as a general method [27-29]. In this assay, extracts were used in a redox-linked reaction whereby the antioxidants presented in the sample act as the oxidants. Reduction of the ferric-tripyridyltriazine to the ferrous complex forms an intense blue colour which can be measured at a wavelength of $593 \mathrm{~nm}$. The intensity of the colour is related to the amount of antioxidant reductants in the extracts. The trend for ferric ion-reducing activities of different fractions from E. ciliata in the present study is shown in Figure 5. The absorbance clearly increased due to the formation of the $\mathrm{Fe}^{2+}$-TPTZ complex with increasing concentration. The higher reducing activity was for the fraction B and E, compared to those of the other fractions (Figure 5). Similar to the results obtained from the DPPH and reducing power assay, the fraction D showed relatively strong ferric ion-reducing activity, while the fractions $\mathrm{A}$ and $\mathrm{C}$ showed lower ferric ion-reducing activities. 
Figure 5. Ferric-reducing antioxidant power (FRAP) assay for fractions of different parts of E. ciliata.
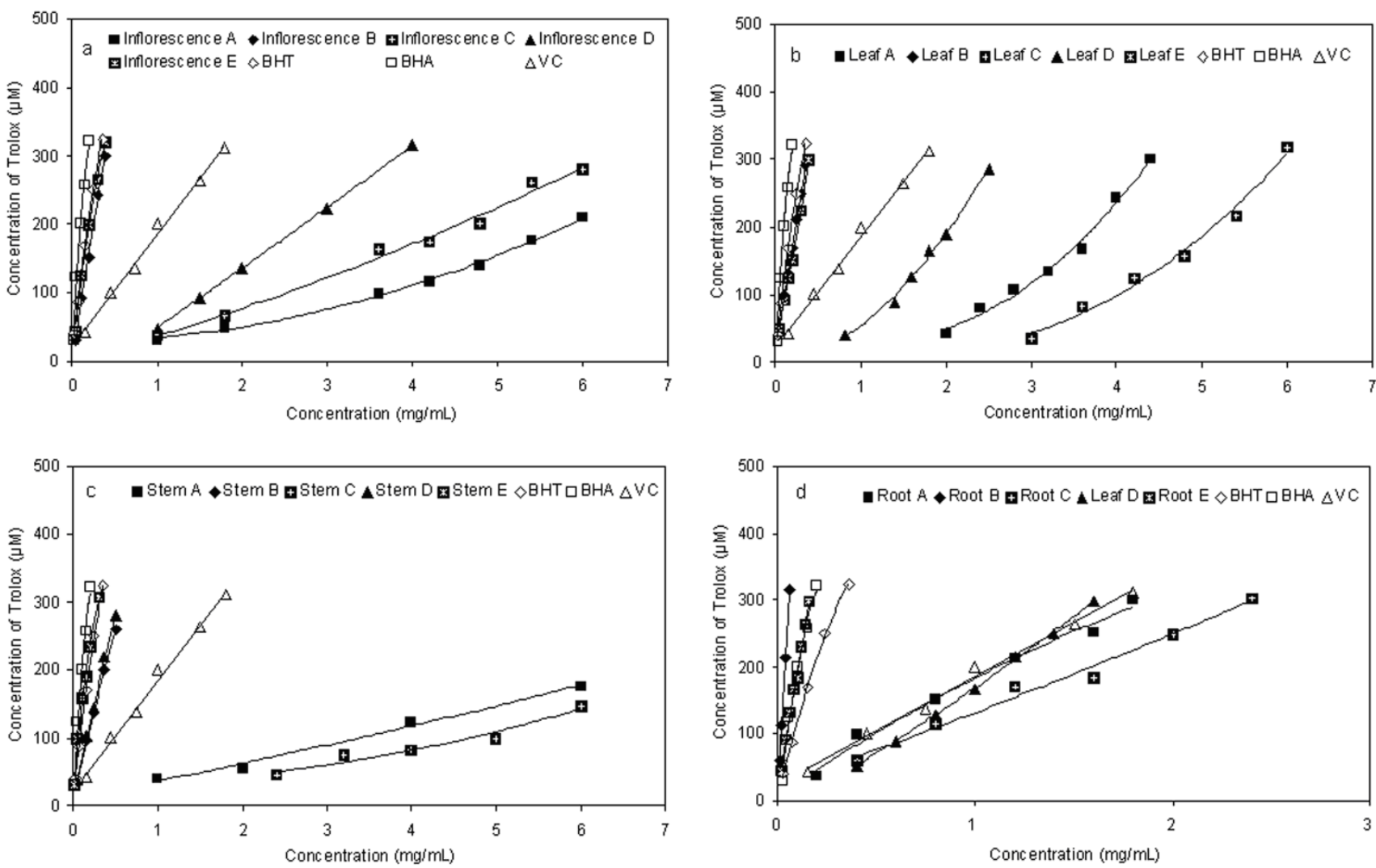

\section{Experimental Section}

\subsection{Plant Materials}

The plant E. ciliata (Labiatae) was collected from the Botanical Garden of Northeast Forestry University and authenticated by Prof. Xiaoying Yuan from the State Engineering Laboratory for Bioresources Eco-Utilization, Northeast Forestry University, China. A specimen was deposited in the Herbarium of this Key Laboratory. The plant samples, including inflorescence, leaf, stem and root, were washed and dried in the shade at room temperature. The dried plants were powdered using a disintegrator.

\subsection{Chemicals}

Folin-Ciocalteu's reagent, 1,1-diphenyl-2-picrylhydrazyl (DPPH), 2,4,6-tripyridyl-S-trizine (TPTZ), 6-hydroxy-2,5,7,8-tetramethylchromane-2-carboxylic acid (Trolox), gallic acid, BHT, BHA, and vitamin $\mathrm{C}\left(\mathrm{V}_{\mathrm{C}}\right)$ were purchased from Sigma-Aldrich Chemical Co. (St. Louis, MO, USA). HPD300 macroporous resin was purchased from Cangzhou Bon Adsorber Technology Co., Ltd. (Hebei, China), the surface area $800-870 \mathrm{~m}^{2} / \mathrm{g}$, the average pore diameter $50-55 \mathrm{~nm}$, the moisture content $75.52 \%$. Prior to the experiments, weighed amounts of resins were soaked in ethanol and subsequently washed by deionized water thoroughly. Then the resins were pretreated by $1 \mathrm{M}$ hydrochloric acid and $1 \mathrm{M}$ sodium hydroxide solution successively to remove the monomers and porogenic agents trapped inside 
the pores during the synthesis process and washed by deionized water until neutral. All other unlabelled chemicals were of analytical grade and were obtained from Beijing Chemical Reagents Co. (Beijing, China). Reverse osmosis Milli-Q water (Millipore, Bedford, MA, USA) was used for all solutions and dilutions.

\subsection{Preparation of the Crude Extract and Fractionation}

$100 \mathrm{~g}$ of the ground dried E. ciliata sample (inflorescence, leaf, stem and root separately) was mixed with $2 \mathrm{~L}$ of water and submitted for $3 \mathrm{~h}$ to hydrodistillation, using a Clevenger-type apparatus. The obtained essential oil was dried over anhydrous sodium sulphate for other purposes. After completion of hydrodistillation, the liquid retentate was decanted, filtered through Whatman No. 2 filter paper (Whatman International Limited, Kent, England) and concentrated in a vacuum evaporator (R206B2, Senco Technology Co. Ltd., Shanghai, China) at $60{ }^{\circ} \mathrm{C}$ and then lyophilized in a freeze-dryer (FD-1, Beijing Boyikang Lab Instrument Co. Ltd., Beijing, China) to obtain a crude water extract A. $5 \mathrm{~g}$ of $\mathrm{A}$ was redissolved in $0.5 \mathrm{~L}$ of distilled water. The solution was consecutively portioned in a separatory funnel with the equivalent amount of ethyl acetate. The ethyl acetate fraction was concentrated in a vacuum evaporator at $40^{\circ} \mathrm{C}$ and then lyophilized in a freeze-dryer to obtain the ethyl acetate fraction B. The aqueous residue was frozen and lyophilized to obtain the fraction C. $1 \mathrm{~g}$ of $\mathrm{B}$ and $\mathrm{C}$ were redissolved in $1 \mathrm{~L}$ of distilled water, respectively, the solution was applied to column chromatography on macroporous resin $(2 \mathrm{~cm} \times 20 \mathrm{~cm})$ with an isocratic elution $80 \%$ ethanol. The ethanol solution was concentrated in a vacuum evaporator at $60{ }^{\circ} \mathrm{C}$ and then lyophilized to obtain the fractions D and E (Figure 1). All the five fractions were stored at $4{ }^{\circ} \mathrm{C}$ for subsequent analyses.

\subsection{Determination of Total Phenolics}

The total phenolics were determined by Folin-Ciocalteu method [30] with little modification. In brief, $200 \mu \mathrm{L}$ of samples $(10 \mathrm{mg} / \mathrm{mL}$ in water) and $1.0 \mathrm{~mL}$ of Folin-Ciocalteu reagent (1:1 with water) were mixed. After $4 \mathrm{~min}, 1.5 \mathrm{~mL}$ of $20 \%$ sodium carbonate was added and the volume was accurately adjusted to $25 \mathrm{~mL}$ with distilled water. The absorbance of the resulting blue color was measured at $765 \mathrm{~nm}$ with a UV-vis spectrophotometer (Shimadzu UV-2550; Shimadzu, Kyoto, Japan) after incubation for $60 \mathrm{~min}$ at room temperature. The determination was performed in triplicate. Quantification was done on the basis of the standard curve of gallic acid. Results were expressed in $g$ gallic acid equivalent (GAE)/g extracts.

\subsection{DPPH free Radical-Scavenging Assay}

DPPH radical-scavenging activity was determined by the method of [31] with some modifications. Briefly, a $3.8 \mathrm{~mL}$ aliquot of freshly prepared DPPH methanol solution $(25 \mu \mathrm{g} / \mathrm{mL})$ was added to $0.2 \mathrm{~mL}$ sample solution at different concentrations. The mixture was shaken vigorously and allowed to stand for $30 \mathrm{~min}$ in the dark. Then the absorbance was measured at $517 \mathrm{~nm}$ in a spectrophotometer. The procedure was performed for different concentrations of each extract. Scavenging activity (SC\%) of free radical DPPH in percent (\%) was calculated in following way:

$$
\mathrm{SC} \%=100 \times\left(A_{\text {blank }}-A_{\text {sample }}\right) / A_{\text {blank }}
$$


where $A_{\text {blank }}$ is the absorbance of the control reaction (containing all reagents except for the test compound), and $A_{\text {sample }}$ is the absorbance of the test compound. $\mathrm{EC}_{50}$ value $(\mu \mathrm{g} / \mathrm{mL})$ is the effective concentration at which DPPH radicals were scavenged by $50 \%$ and was obtained by interpolation from regression analysis from the graph plotting inhibition percentage against extract concentration. BHA, BHT and Vc were used as positive control. Tests were carried out in triplicate.

\subsection{Measurement of Reducing Power}

The reducing power was determined as described by [25], but with slight modifications. Briefly, $1 \mathrm{~mL}$ each of extracts at different concentrations was mixed with $2.5 \mathrm{~mL}$ of $0.2 \mathrm{~mol} / \mathrm{L}$ phosphate buffer $\left(\mathrm{pH}\right.$ 6.6) and $2.5 \mathrm{~mL}$ of potassium ferricyanide (1\%). The mixture was then incubated at $50{ }^{\circ} \mathrm{C}$ for $20 \mathrm{~min}$. Then, $2.5 \mathrm{~mL}$ of $10 \%$ trichloroacetic acid (TCA) was added, and the mixture was then centrifuged at 3,000 g for $10 \mathrm{~min}$. The upper layer $(2.5 \mathrm{~mL})$ was mixed with $2.5 \mathrm{~mL}$ distilled water and $0.5 \mathrm{~mL}$ of $0.1 \%$ ferric chloride, and the absorbance was measured at $700 \mathrm{~nm}$ in a spectrophotometer. Increased absorbance of the reaction mixture indicated higher reducing power. BHA, BHT and Vc were used as positive control. All determinations were performed in triplicate.

\subsection{Total Antioxidant Capacity by FRAP Assay}

The FRAP assay was carried out according to the procedure of [32] with slight modifications. Briefly, the FRAP reagent was prepared from $300 \mathrm{mM}$ sodium acetate buffer (pH 3.6), $10 \mathrm{mM} \mathrm{TPTZ}$ solution in $40 \mathrm{mM} \mathrm{HCl}$ and $20 \mathrm{mM} \mathrm{FeCl}_{3}$ solution in proportions of 10:1:1 (v/v), respectively. The FRAP reagent was prepared fresh daily and was warmed to $37{ }^{\circ} \mathrm{C}$ in a water bath prior to use. A $150 \mu \mathrm{L}$ of sample solution of different concentrations was added to $2.85 \mathrm{~mL}$ of FRAP reagent and then standed for $30 \mathrm{~min}$ in the dark. The absorbance of the reaction extract solution was recorded at $593 \mathrm{~nm}$. The standard curve was constructed using Trolox solution $(37.5-600 \mu \mathrm{M})$. In the FRAP assay, the antioxidant efficiency of the antioxidant under the test was calculated with reference to the reaction signal given by Trolox solution of known concentration. The results were corrected for dilution and expressed in $\mu \mathrm{mol}$ Trolox/L. BHA, BHT and Vc were used as positive controls. The assay was carried out in triplicate.

\subsection{Statistical Analysis}

All data were given as means \pm SD. One-way analysis of variance (ANOVA) and Duncan's multiple range test were carried out to determine significant differences $(p<0.05)$ between the means by SPSS (version 10.1).

\section{Conclusions}

This study was designed to evaluate the antioxidant and free radical scavenging activities of extract and fractions from E. ciliata using three in vitro antioxidant models. Fraction root E, showed the highest total phenolic contents, and followed by fraction root $\mathrm{B}$, fraction stem $\mathrm{E}$ and fraction inflorescence $\mathrm{E}$ in turn. Meanwhile, fraction root $\mathrm{E}$ exhibited higher antioxidant and free-radical scavenging activities, which are stronger than the positive control BHT, BHA and $\mathrm{V}_{\mathrm{C}}$. 
The two enrichment methods of phenolic components: ethyl acetate-water liquid-liquid extraction and macroporous resin adsorption-desorption, were adopted in this study. The macroporous resin technology was effective for enrichment of phenolic components in dealing with ethyl acetate extraction residue and ethyl acetate extract under similar operating parameters.

This study indicated that the extracts from E. ciliata have great potential to prevent diseases caused by the overproduction of radicals. The present investigation may provide the evidence required for the utilization of E. ciliata as a kind of herbal drug as well as the possibility of using E. ciliata as a source of low-cost natural antioxidants. However, further work is still needed to identify and characterise the inherent phytocompounds from relational fractions and to investigate the antioxidant efficacy of E. ciliata in vivo.

\section{Acknowledgments}

The authors thank anonymous reviewers for their insightful comments and careful corrections. The authors also thank the Special Fund for Forestry Scientific Research in the Public Interest (201204601) and the National Key Technology R\&D Program (2012BAD21B05) for financial support.

\section{References and Notes}

1. Finkel, T.; Holbrook, N.J. Oxidants, oxidative stress and the biology of ageing. Nature 2000, 408, 239-247.

2. Gülçin, İ. Antioxidant activity of food constituents: An overview. Arch. Toxicol. 2012, 86, 345-396.

3. Bursal, E.; Gülçin, İ. Polyphenol contents and in vitro antioxidant activities of lyophilized aqueous extract of kiwifruit (Actinidia deliciosa). Food Res. Int. 2011, 44, 1482-1489.

4. Cornwell, D.G.; Jones, K.H.; Jiang, Z.; Lantry, L.E.; Southwell-Keely, P.; Kohar, I.; Thornton, D.E. Cytotoxicity of tocopherols and their quinones in drug-sensitive and multidrug-resistant leukemia cells. Lipids 1998, 33, 295-301.

5. Namiki, M. Antioxidants / antimutagens in food. Crit. Rev. Food Sci. 1990, 29, 273-300.

6. Namiki, M.; Osawa, T. Antioxidants / antimutagens in foods. Basic Life Sci. 1986, 39, 131-142.

7. Stich, H.F. The beneficial and hazardous effects of simple phenolic compounds. Mutat. Res-Gen. Tox. 1991, 259, 307-324.

8. Kouri, G.; Tsimogiannis, D.; Bardouki, H.; Oreopoulou, V. Extraction and analysis of antioxidant components from Origanum dictamnus. Innov. Food Sci. Emerg. Tech. 2007, 8, 155-162.

9. Oke, F.; Aslim, B.; Ozturk, S.; Altundag, S. Essential oil composition, antimicrobial and antioxidant activities of Satureja cuneifolia Ten. Food Chem. 2009, 112, 874-879.

10. Politeo, O.; Jukic, M.; Milos, M. Chemical composition and antioxidant capacity of free volatile aglycones from basil (Ocimum basilicum L.) compared with its essential oil. Food Chem. 2007, 101, 379-385.

11. Wang, S.; Ballington, J. Free radical scavenging capacity and antioxidant enzyme activity in deerberry (Vaccinium stamineum L.). LWT-Food Sci. Tech. 2007, 40, 1352-1361.

12. Yang, J.; Guo, J.; Yuan, J. In vitro antioxidant properties of rutin. LWT-Food Sci. Tech. 2008, 41, 1060-1066. 
13. Jia, Z.; Tao, F.; Guo, L.; Tao, G.; Ding, X. Antioxidant properties of extracts from juemingzi (Cassia tora L.) evaluated in vitro. LWT-Food Sci. Tech. 2007, 40, 1072-1077.

14. Melkani, A.; Dev, V.; Beauchamp, P.; Negi, A.; Mehta, S.; Melkani, K. Constituents of the essential oil of a new chemotype of Benth. Biochem. Syst. Ecol. 2005, 33, 419-425.

15. Peng, H.Y.; Yang, X.E. Volatile constituents in the flowers of Elsholtzia argyi and their variation: A possible utilization of plant resources after phytoremediation. J. Zhejiang Univ. Sci. B 2005, 6, 91-95.

16. She, G.; Guo, Z.; Lv, H.; She, D. New flavonoid glycosides from Elsholtzia rugulosa Hemsl. Molecules 2009, 14, 4190-4196.

17. Liu, A.L.; Liu, B.; Qin, H.L.; Lee, S.; Wang, Y.T.; Du, G.H. Anti-influenza virus activities of flavonoids from the medicinal plant Elsholtzia rugulosa. Planta Med. 2008, 74, 847-851.

18. Zheng, S.; Lü, R.; Shen, X. Studies on the flavonoids of Elsholtzia ciliata Hyland. Chem. J. Chin. Univ. 1989, 10, 866-868.

19. Kim, H.H.; Yoo, J.S.; Lee, H.S.; Kwon, T.K.; Shin, T.Y.; Kim, S.H. Elsholtzia ciliata inhibits mast cell-mediated allergic inflammation: Role of calcium, p38 mitogen-activated protein kinase and nuclear factor-кB. Exp. Biol. Med. 2011, 236, 1070-1077.

20 Hayase, F.; Kato, H. Antioxidative components of sweet potatoes. J. Nutr. Sci. Vitaminol. 1984, 30, 37-46.

21. Kedage, V.V.; Tilak, J.C.; Dixit, G.B.; Devasagayam, T.P.; Mhatre, M. A study of antioxidant properties of some varieties of grapes (Vitis vinifera L.). Crit. Rev. Food Sci. Nutr. 2007, 47, $175-185$.

22 Sarikurkcu, C.; Tepe, B.; Daferera, D.; Polissiou, M.; Harmandar, M. Studies on the antioxidant activity of the essential oil and methanol extract of Marrubium globosum subsp. globosum (lamiaceae) by three different chemical assays. Bioresource Technol. 2008, 99, 4239-4246.

23. Gülçin, İ. Antioxidant activity of eugenol: A structure and activity relationship study. J. Med. Food 2011, 14, 975-985.

24. Gülçin, İ.; Topal, F.; Çakmakçı, R.; Bilsel, M.; Gören, A.C.; Erdogan, U. Pomological features, nutritional quality, polyphenol content analysis and antioxidant properties of domesticated and three wild ecotype forms of raspberries (Rubus idaeus L.). J. Food Sci. 2011, 76, C585-C593.

25. Liu, J.; Wang, C.; Wang, Z.; Zhang, C.; Lu, S.; Liu, J. The antioxidant and free-radical scavenging activities of extract and fractions from corn silk (Zea mays L.) and related flavone glycosides. Food Chem. 2011, 126, 261-269.

26. Gülçin, I.; Bursal, E.; Sehitoğlu, M.H.; Bilsel, M.; Gören, A.C. Polyphenol contents and antioxidant activity of lyophilized aqueous extract of propolis from Erzurum, Turkey. Food Chem. Toxicol. 2010, 48, 2227-2238.

27. Aaby, K.; Wrolstad, R.E.; Ekeberg, D.; Skrede, G. Polyphenol composition and antioxidant activity in strawberry purees; impact of achene level and storage. J. Agric. Food Chem. 2007, 55, 5156-5166.

28. Asadi, S.; Khodagholi, F.; Esmaeili, M.A.; Tusi, S.K.; Ansari, N.; Shaerzadeh, F.; Sonboli, A.; Ghahremanzamaneh, M. Chemical composition analysis, antioxidant, antiglycating activities and neuroprotective effects of $S$. choloroleuca, S. mirzayanii and S. santolinifolia from Iran. Am. J. Chin. Med. 2011, 39, 615-638. 
29 Bakasso, S.; Lamien-Meda, A.; Lamien, C.E.; Kiendrebeogo, M.; Millogo, J.; Ouedraogo, A.G.; Nacoulma, O.G. Polyphenol contents and antioxidant activities of five Indigofera species (Fabaceae) from Burkina Faso. Pakistan J. Biol. Sci. 2008, 11, 1429-1435.

30. Su, L.; Yin, J.J.; Charles, D.; Zhou, K.; Moore, J.; Yu, L. Total phenolic contents, chelating capacities, and radical-scavenging properties of black peppercorn, nutmeg, rosehip, cinnamon and oregano leaf. Food Chem. 2007, 100, 990-997.

31. Yang, L.; Huang, J.M.; Zu, Y.G.; Ma, C.H.; Wang, H.; Sun, X.W.; Sun, Z. Preparation and radical scavenging activities of polymeric procyanidins nanoparticles by a supercritical antisolvent (SAS) process. Food Chem. 2011, 128, 1152-1159.

32. Li, H.; Wong, C.; Cheng, K.; Chen, F. Antioxidant properties in vitro and total phenolic contents in methanol extracts from medicinal plants. LWT-Food Sci. Tech. 2008, 41, 385-390.

Sample Availability: Samples of the compounds are available from the authors.

(C) 2012 by the authors; licensee MDPI, Basel, Switzerland. This article is an open access article distributed under the terms and conditions of the Creative Commons Attribution license (http://creativecommons.org/licenses/by/3.0/). 